\title{
Fisioterapia Complexa Descongestiva Associada a Terapias de Compressão no Tratamento do Linfedema Secundário ao Câncer de Mama: uma Revisão Sistemática
}

Complex Decongestive Physiotherapy Associated Compression Therapy in the Treatment of Secondary Lymphedema in Breast Cancer: a Systematic Review Fisioterapia Compleja Descongestiva Asociada a Terapia de Compresión en el Tratamiento del Linfedema Secundario al Cáncer de Mama: una Revisión Sistemática

Suiane Weimer Cendron'; Luciana Laureano Paiva ${ }^{2}$ Caroline Darski ${ }^{3}$ Cássia Colla ${ }^{4}$

\section{Resumo}

Introdução: $\mathrm{O}$ câncer de mama é a neoplasia mais incidente na população feminina, sendo o linfedema de membro superior a complicação mais frequente no pós-operatório de mastectomia. Objetivo: Verificar a eficácia da fisioterapia complexa descongestiva associada ao uso de kinesiotape, enfaixamento compressivo e compressão pneumática no tratamento do linfedema secundário ao câncer de mama. Método: Revisão sistemática, cujas bases de dados consultadas foram: MEDLINE, PEDro, Cochrane CENTRAL, EMBASE, Periódicos Capes, Cinahl e Scholar Google. As palavras-chave foram: "kinesiotape”, "bandage”, "drainage", "mastectomy”, "lymphedema”, "physioterapy”, "physical therapy", "compressive bandaging", "pneumatic compression". Resultados: Foram selecionados dez ensaios clínicos randomizados. Foi possível verificar que a compressão pneumática reduz os níveis de volume de linfedema quando associada à fisioterapia complexa descongestiva. O enfaixamento compressivo se mostra eficaz na redução do linfedema; porém muitas vezes é abandonado pelas pacientes pelo desconforto que causa. A kinesiotape surge como uma alternativa terapêutica para pacientes que não se adaptaram ao enfaixamento; porém, os artigos que abordam essa temática são em sua maioria estudos de casos e, por isso, se tornam insuficientes para confirmar sua eficácia. Conclusão: Essa revisão sistemática mostrou-se relevante na medida em que permitiu verificar os efeitos de cada uma das técnicas no tratamento do linfedema pós-mastectomia. No entanto, os estudos clínicos randomizados ainda se mostram escassos, principalmente no que se refere ao uso da kinesiotape. Por essa razão, não há como estabelecer qual técnica é mais eficaz na redução do linfedema e na manutenção dos níveis de redução conquistados durante a fisioterapia complexa descongestiva.

Palavras-chave: Mastectomia; Linfedema; Fisioterapia; Bandagens Compressivas

\footnotetext{
${ }^{1}$ Fisioterapeuta. Graduada pela Universidade Federal do Rio Grande do Sul (UFRGS). Porto Alegre (RS), Brasil. E-mail: suiwcendron@gmail.com.

${ }^{2}$ Fisioterapeuta. Professora Doutora do Curso de Fisioterapia da UFRGS. Porto Alegre (RS), Brasil. E-mail: lucianalaureanopaiva@gmail.com.

${ }^{3}$ Fisioterapeuta. Graduada pela UFRGS. Porto Alegre (RS), Brasil. E-mail: carolzinha_darski@hotmail.com.

${ }^{4}$ Fisioterapeuta. Graduada pela UFRGS. Porto Alegre (RS), Brasil. E-mail: cassia.colla1@gmail.com.

Endereço para correspondência: Suiane Weimer Cendron. Rua Florianópolis, 1.315 - Canoas (RS), Brasil. CEP: 92330-500.
} 


\section{INTRODUÇÃO}

O câncer de mama é atualmente o tipo de câncer com maior índice de óbito e incidência na população feminina ${ }^{1}$. Independente da abordagem cirúrgica utilizada no seu tratamento, inúmeras complicaçôes podem ocorrer no período pós-operatório, produzindo sintomas dolorosos e incapacitantes, interferindo no processo de recuperação ${ }^{2}$.

Entre as alterações presentes, o linfedema surge como uma das complicaçôes mais comuns após a cirurgia da mama $^{3}$, ocasionado geralmente pelo esvaziamento dos linfonodos axilares. Os principais sinais e sintomas presentes são: redução da funcionalidade e aumento do diâmetro do membro homolateral à cirurgia, rigidez e diminuição da amplitude de movimento do membro acometido e distúrbios sensoriais ${ }^{4}$.

No tratamento do linfedema, a fisioterapia ocupa lugar de destaque ${ }^{5}$, podendo ser realizada em duas fases: a intensiva e a de manutenção $0^{4,6}$. A primeira fase é composta pela fisioterapia complexa descongestiva (FCD), técnica que combina drenagem linfática manual (DLM) com os seguintes procedimentos: enfaixamento compressivo funcional (ECF), kinesiotape (K-TAPE), contenção elástica, compressão pneumática intermitente (CPI), exercícios terapêuticos, cuidados com a pele e cuidados na vida diária ${ }^{4-6}$. Já na fase de manutenção, os recursos mais aplicados são a automassagem linfática, os exercícios funcionais, uso de contenção elástica e cuidados com a pele ${ }^{4,6}$.

A compressão externa é definida como a aplicação de qualquer pressão externa no membro com o intuito de reduzir a formação de edema e auxiliar a remoção do excesso de fluido linfático já acumulado sendo importante para a drenagem linfática, pois a pressão gerada irá promover um diferencial de pressão entre as extremidades deslocando o fluido contido no vaso linfático, promovendo uma redução da pressão do seu interior e, assim, facilitando a entrada do excesso de líquido contido no interstício para o vaso por diferença pressórica, podendo ser realizada por meio de uma variedade de técnicas, como, por exemplo, o ECF, a K-TAPE ou a CPI ${ }^{6-9}$.

Devido à escassez de revisôes sistemáticas na literatura, que busquem elucidar qual a melhor terapia para a reduçáo do linfedema, se faz importante investigar qual terapia promove maior reduçáo do linfedema de membro superior para que se possa embasar a prática clínica em evidência científica.

Dessa forma, o presente estudo se propóe a realizar uma revisão sistemática com o intuito de verificar a eficácia da FCD associada ao uso de enfaixamento compressivo (EC), K-TAPE ou compressáo pneumática no tratamento do linfedema de membro superior de mulheres mastectomizadas.

\section{MÉTODO}

A presente revisão segue as diretrizes PRISMA (Preferred Reporting Items for Systematic Review and Metaanalyses $)^{10}$.

\section{ESTRATÉGIA DE BUSCA}

A busca de artigos foi realizada no período de março a outubro de 2013, nos seguintes bancos de dados eletrônicos: MEDLINE (acessado via PubMed), Physiotherapy Evidence Database (PEDro), Register of Controlled Trials (Cochrane CENTRAL), EMBASE, Periódicos Capes, Cinahl e Scholar Google. Admitiram-se estudos publicados a partir do ano de 2000.

A estratégia de busca utilizou os seguintes termos: "kinesiotape", "bandage", "drainage", "mastectomy", "lymphedema", "physioterapy", "physical therapy", "compressive bandaging", "pneumatic compression", sendo que os mesmos termos foram utilizados também para pesquisa em português. Na base PubMed, foram utilizados os termos meshs correspondentes. As combinaçóes entre as palavras-chave foram realizadas em cada base de dados utilizando os operadores booleanos $O R$, AND e NOT AND. A pesquisa incluiu artigos em português, inglês e espanhol.

\section{CRITÉRIOS DE ELEGIBILIDADE}

Foram excluídos artigos de revisão e estudos de caso, ou estudos que utilizaram a FCD associada à laserterapia ou estimulação elétrica, além de estudos pilotos que já possuíam estudo completo publicado.

\section{SELEÇÃO DOS ESTUDOS E EXTRAÇÃO DOS DADOS}

Os estudos foram selecionados conforme conteúdo do título e resumo, por dois avaliadores independentes. Os artigos que estavam de acordo com os critérios estabelecidos, aprovados por ambos avaliadores, foram incluídos na revisão sistemática. As discordâncias entre os avaliadores foram resolvidas por consenso.

Para a extração dos dados, selecionou-se, como desfecho principal, a redução no volume do membro superior afetado, avaliado por deslocamento de água e circunferência; e, como desfecho secundário, foram extraídos sintomas subjetivos como redução na sensação de peso, dor e inchaço no membro.

\section{AVALIAÇÃO DA QUALIDADE DOS ESTUDOS}

Os ensaios clínicos randomizados (ECR) foram verificados quanto à qualidade metodológica conforme a escala PEDro. Essa escala avalia as seguintes questôes: 1) critérios de eligibilidade; 2) distribuiçáo aleatória; 3 ) distribuiçáo cega; 4) diferenças entre os grupos no baseline; 5) participação cega; 6) intervenção cega; 7) avaliação cega; 8) resultados com mais de $85 \%$ da amostra; 9) 
situação controle; 10) resultados intergrupos; 11) medidas de precisáo. $\mathrm{O}$ escore da escala varia de 0 a 10 pontos. Um ponto é concedido a cada um dos 11 critérios, se satisfeito, não se pontuando o primeiro item. Os estudos randomizados e controlados de boa qualidade foram definidos com escores variando de seis a oito pontos, de qualidade moderada variando de quatro a cinco pontos, e de qualidade pobre variando de três pontos ou menos na escala PEDro.

\section{RESULTADOS}

A pesquisa inicial nas bases de dados encontrou 3.010 artigos e, após uma primeira seleção por título, foram excluídos 2.942 artigos, restando 68 para análise dos resumos. Destes, foram selecionados 24 artigos. Após leitura completa desses estudos, eliminaram-se mais 14 artigos que não se enquadravam nos critérios de inclusão estabelecidos (Figura 1).

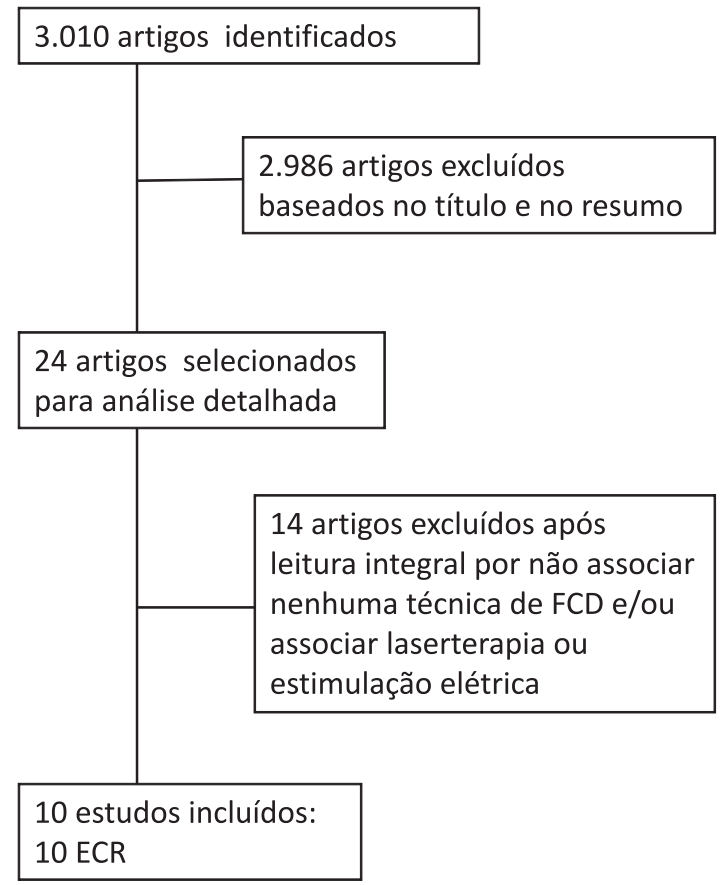

Figura 1. Fluxograma de busca eletrônica e seleção de estudos

Dos dez artigos incluídos ao final da busca, todos apresentavam delineamento do tipo ECR, totalizando 444 participantes, com idades variando de 28 a 89 anos, sem grupos controle.

Quanto à avaliação metodológica dos ensaios clínicos randomizados, nenhum estudo foi considerado de baixa qualidade. Os estudos incluídos nesta revisão foram considerados com baixo risco de viés (Figura 2). Nenhum dos artigos avaliados apresentou descrito em seu texto o cálculo amostral.

\begin{tabular}{|cccccccccccc|}
\hline Estudo / Ano & 2 & 3 & 4 & 5 & 6 & 7 & 8 & 9 & 10 & $\mathbf{1 1}$ & $\begin{array}{c}\text { Escore } \\
\text { Final }\end{array}$ \\
$\begin{array}{c}\text { Szolnoky et al } \\
(2010)\end{array}$ & $\mathrm{S}$ & $\mathrm{NI}$ & $\mathrm{NI}$ & $\mathrm{NI}$ & $\mathrm{NI}$ & $\mathrm{NI}$ & $\mathrm{S}$ & $\mathrm{S}$ & $\mathrm{S}$ & $\mathrm{S}$ & 5 \\
$\begin{array}{c}\text { Haghighat et al } \\
(2010)\end{array}$ & $\mathrm{S}$ & $\mathrm{NI}$ & $\mathrm{S}$ & $\mathrm{NI}$ & $\mathrm{S}$ & $\mathrm{S}$ & $\mathrm{S}$ & $\mathrm{S}$ & $\mathrm{S}$ & $\mathrm{S}$ & 8 \\
$\begin{array}{c}\text { Fife et al } \\
(2012)\end{array}$ & $\mathrm{S}$ & $\mathrm{S}$ & $\mathrm{NI}$ & $\mathrm{NI}$ & $\mathrm{NI}$ & $\mathrm{S}$ & $\mathrm{S}$ & $\mathrm{S}$ & $\mathrm{S}$ & $\mathrm{S}$ & 7 \\
$\begin{array}{c}\text { Gurdal et al } \\
(2012)\end{array}$ & $\mathrm{S}$ & $\mathrm{S}$ & $\mathrm{N}$ & $\mathrm{N}$ & $\mathrm{N}$ & $\mathrm{S}$ & $\mathrm{S}$ & $\mathrm{S}$ & $\mathrm{S}$ & $\mathrm{S}$ & 7 \\
$\begin{array}{c}\text { Uzkeser et al } \\
(2013)\end{array}$ & $\mathrm{S}$ & $\mathrm{S}$ & $\mathrm{S}$ & $\mathrm{S}$ & $\mathrm{S}$ & $\mathrm{S}$ & $\mathrm{S}$ & $\mathrm{S}$ & $\mathrm{S}$ & $\mathrm{S}$ & 10 \\
$\begin{array}{c}\text { Mnellly et al } \\
(2004)\end{array}$ & $\mathrm{S}$ & $\mathrm{S}$ & $\mathrm{NI}$ & $\mathrm{NI}$ & $\mathrm{S}$ & $\mathrm{S}$ & $\mathrm{S}$ & $\mathrm{S}$ & $\mathrm{S}$ & $\mathrm{S}$ & 8 \\
$\begin{array}{c}\text { Damstra et al } \\
(2009)\end{array}$ & $\mathrm{S}$ & $\mathrm{S}$ & $\mathrm{NI}$ & $\mathrm{NI}$ & $\mathrm{NI}$ & $\mathrm{NI}$ & $\mathrm{S}$ & $\mathrm{S}$ & $\mathrm{S}$ & $\mathrm{S}$ & 6 \\
$\begin{array}{c}\text { Kasseroller et al } \\
(2009)\end{array}$ & $\mathrm{S}$ & $\mathrm{S}$ & $\mathrm{NI}$ & $\mathrm{NI}$ & $\mathrm{NI}$ & $\mathrm{NI}$ & $\mathrm{S}$ & $\mathrm{S}$ & $\mathrm{S}$ & $\mathrm{S}$ & 6 \\
$\begin{array}{c}\text { King et al } \\
(2011)\end{array}$ & $\mathrm{S}$ & $\mathrm{NI}$ & $\mathrm{NI}$ & $\mathrm{N}$ & $\mathrm{S}$ & $\mathrm{S}$ & $\mathrm{S}$ & $\mathrm{S}$ & $\mathrm{S}$ & $\mathrm{NI}$ & 6 \\
$\quad \begin{array}{c}\text { Tsai et al } \\
(2009)\end{array}$ & $\mathrm{S}$ & $\mathrm{S}$ & $\mathrm{NI}$ & $\mathrm{S}$ & $\mathrm{N}$ & $\mathrm{S}$ & $\mathrm{S}$ & $\mathrm{S}$ & $\mathrm{S}$ & $\mathrm{S}$ & 8 \\
\hline
\end{tabular}

Figura 2. Escala de Avaliação da Qualidade Metodológica (PEDro)

\section{FISIOTERAPIA COMPLEXA DESCONGESTIVA ASSOCIADA À COMPRESSÃO PNEUMÁTICA INTERMITENTE}

Para verificar a eficácia da FCD associada ao uso da CPI no tratamento do linfedema de membro superior pós-mastectomia, foram incluídos cinco estudos, com delineamento do tipo ensaio clínico randomizado (Quadro1).

Szolnoky et al. (2009) ${ }^{11}$ investigaram se a CPI associada à DLM poderia melhorar os resultados da FCD no tratamento de mulheres com linfedema secundário após tratamento do câncer de mama comparando com a aplicação da DLM isolada. Após avaliar e tratar 27sujeitos, verificou-se que a DLM isoladamente ou em conjunto com a CPI, como parte de um protocolo de FCD, resultou em reduçôes notáveis no linfedema de membro superior e nos sintomas relatados pelos participantes, como dor e sensação de peso. Para análise estatística, os autores utilizaram testes $t$ de Student e as diferenças de volume foram consideradas significativas quando $\mathrm{p}<0,05$.

Já Haghighat et al. $(2010)^{12}$, diferente do estudo anterior, verificaram, ao comparar dois métodos de tratamento para o linfedema de membro superior pós-mastectomia, que tanto a Terapia Complexa Descongestiva (TCD) como a TCD Modificada (associada à compressão pneumática) promovem redução no volume do membro afetado, no entanto a redução foi mais expressiva no grupo que realizou a TCD sem adição de CPI. A Diferença entre os dois grupos foi estudada pelo teste $t$ de Student, enquanto o teste de Mann-Whitney foi usado para mostrar as variaçôes de sintomas dos grupos. A significânia estatística foi fixada quando encontrados valores de $\mathrm{p}<0,05$.

Fife et al. $(2012)^{13}$ avaliaram 36 mulheres e compararam as mudanças no volume de edema no membro superior obtidas com a utilização de um sistema de compressão pneumática avançada (compressão em membro superior, tronco e peito) com um sistema de $\mathrm{CP}$ padrão. Utilizando 
teste $t$ de Student para amostras independentes na análise estatística e considerando como diferença significativa valores de $\mathrm{p}<0,05$, chegaram à conclusão de que ambas as terapias reduzem o volume, sendo mais expressiva no grupo que utilizou o sistema avançado.

Gurdal et al. $(2012)^{14}$ realizaram um estudo com 30 sujeitos comparando a DLM combinada ao ECF com CPI e verificaram que ambas as terapias diminuem o volume do membro afetado, não apresentando diferença significativa entre os grupos. Os autores utilizaram-se dos testes de $t$ de Student, Mann-Whitney-U e Wilcoxon na análise estatística, e os valores foram considerados significantes quando $\mathrm{p}<0,001$.

Por fim, Uzkeser et al. (2013) 15 investigaram a eficácia e a contribuição da CPI no tratamento do linfedema de membro superior e chegaram à conclusão de que a CPI náo promove maiores ganhos no tratamento do linfedema quando comparada com a FCD padráo. Foram utilizados os testes Mann-Whitney e qui-quadrado para comparar as variáveis entre os dois grupos. O teste de Wilcoxon foi utilizado para avaliar os valores nos pré e pós-tratamentos dentro dos grupos. A análise de correlaçáo entre as medidas dos métodos foi realizada com a correlaçáo de Pearson. $\mathrm{O}$ nível de significância estatística foi estabelecido em $\mathrm{p}<0,05$.

\section{FISIOTERAPIA COMPLEXA DESCONGESTIVA ASSOCIADA AO ENFAIXAMENTO COMPRESSIVO FUNCIONAL}

Foram encontrados quatro estudos do tipo ensaio clínico randomizado que investigaram a associação entre a FCD e o ECF no tratamento do linfedema de membro superior pós-mastectomia (Quadro2).

McNeely et al. $(2004)^{16}$ verificaram a redução do linfedema de membro superior utilizando em um grupo a DLM em combinaçáo com ECF e outro grupo somente o ECF. Foi possível perceber que não houve diferença

Quadro 1. Característica dos estudos clínicos randomizados selecionados sobre fisioterapia complexa descongestiva e compressão pneumática intermitente

\begin{tabular}{|c|c|c|c|c|c|c|}
\hline Estudo/Ano & Objetivo & N & $\begin{array}{c}\text { Média } \\
\text { de idade }\end{array}$ & Tratamento & $\begin{array}{c}\text { Avaliação do } \\
\text { linfedema }\end{array}$ & Resultados obtidos \\
\hline $\begin{array}{l}\text { Szolnoky et } \\
\text { al., } 2009^{11}\end{array}$ & $\begin{array}{l}\text { Investigar se } \\
\text { a compressão } \\
\text { pneumatica } \\
\text { intermitente associada } \\
\text { à DLM poderia } \\
\text { melhorar os resultados } \\
\text { da FCD no tratamento } \\
\text { de mulheres com } \\
\text { linfedema de membro } \\
\text { superior pós- } \\
\text {-mastectomia }\end{array}$ & 27 & $\begin{array}{l}\text { Grupo I: } \\
54,83 \\
\text { Grupo II: } \\
56,6\end{array}$ & $\begin{array}{l}\text { Tratamento: Grupo I: recebeu } 60 \text { min de } \\
\text { drenagem linfática manual } \\
\text { Grupo II: recebeu } 30 \text { min de DLM + } 30 \\
\text { min de compressão pneumática } \\
\text { Além disso, foram realizados em ambos } \\
\text { os grupos cuidados com a pele, EC e } \\
\text { exercícios de membro superior }\end{array}$ & $\begin{array}{l}\text { Perimetria } \\
\text { do membro } \\
\text { superior a cada } \\
4 \mathrm{~cm}\end{array}$ & $\begin{array}{l}\text { No grupo I, o volume reduziu no } \\
\text { final da terapia 3,06\%. Após } 1 \text { e } 2 \\
\text { meses de terapia, a porcentagem de } \\
\text { linfedema reduziu } 2,9 \% \text { e } 3,6 \% \text {, } \\
\text { respectivamente } \\
\text { No grupo II, a redução ao final } \\
\text { da terapia foi de } 7,93 \% \text {. Um mês } \\
\text { após, o grupo apresentou uma } \\
\text { redução de } 9,02 \% \text {; e, após } 2 \\
\text { meses, de } 9,6 \%\end{array}$ \\
\hline $\begin{array}{l}\text { Haghighat et } \\
\text { al., } 2010^{12}\end{array}$ & $\begin{array}{l}\text { Comparar dois } \\
\text { métodos de tratamento } \\
\text { para o linfedema } \\
\text { pós-mastectomia: } \\
\text { TCD e TCD modificada } \\
\text { (MTCD), combinada } \\
\text { com CPI }\end{array}$ & 112 & $\begin{array}{l}\text { Grupo I: } \\
53,4 \\
(+-11,4) \\
\text { Grupo II: } \\
52,7 \\
(+-10,8)\end{array}$ & $\begin{array}{l}\text { Os tratamentos foram implementados em } \\
\text { duas fases: } \\
\text { Fase I (intensiva) } \\
5 x \text { por semana, } 10-15 \text { sessões } \\
\text { Grupo I: Fase I consistiu-se de cuidado } \\
\text { com a pele, } 45 \text { minutos de DLM, exercícios } \\
\text { de reforço, e compressão aplicada pelo EC } \\
\text { Grupo II: } 10-15 \text { min de DLM. Logo após } \\
\text { uma manga de quatro câmaras para CPI } \\
\text { foi fixada em } 40 \text { mmHg a pressão por } \\
30 \text { minutos. A Drenagem linfática do } \\
\text { braço foi concluída com cinco minutos de } \\
\text { DLM. Os outros três componentes do TCD } \\
\text { (cuidados com a pele, exerćícios corretivos } \\
\text { e curativos) foram idênticos ao grupo I } \\
\text { Fase II (fase de manutenção) } \\
\text { Os grupos foram orientados para } \\
\text { autoaplicação de compressão por órtese } \\
\text { elástica durante o dia e EC à noite. } \\
\text { Exerćícios corretivos e cuidados com a } \\
\text { pele se mantiveram os mesmos, além de } \\
\text { automassagem leve (1 ou } 2 x \text { ao dia) }\end{array}$ & $\begin{array}{l}\text { Método de } \\
\text { deslocamento } \\
\text { de água, em } \\
\text { que o membro } \\
\text { superior é } \\
\text { imerso em } \\
\text { um recipiente } \\
\text { com água e } \\
\text { o volume de } \\
\text { água deslocado } \\
\text { é mensurado. } \\
0 \text { edema foi } \\
\text { definido pela } \\
\text { diferença de } \\
\text { deslocamento } \\
\text { de água entre } \\
\text { o membro } \\
\text { superior afełado } \\
\text { e o membro } \\
\text { superior } \\
\text { saudável }\end{array}$ & $\begin{array}{l}\text { Grupo I reduziu } 43,1 \% \text { (+-13,7) } \\
\text { o volume do membro na fase I e } 0 \\
\text { grupo II 37,5\% (+-14,4) } \\
\text { Após } 3 \text { meses de acompanhamento, } \\
\text { houve um adicional de redução de } \\
16,9 \%(+-2,3) \text { para } 0 \text { grupo I e } \\
7,5 \% \text { (+-39,4) para o grupo } 2\end{array}$ \\
\hline
\end{tabular}


Quadro 1. continuação

\begin{tabular}{|c|c|c|c|c|c|c|}
\hline Estudo/Ano & Objetivo & $\mathbf{N}$ & $\begin{array}{c}\text { Média } \\
\text { de idade }\end{array}$ & Tratamento & $\begin{array}{c}\text { Avaliação do } \\
\text { linfedema }\end{array}$ & Resultados obtidos \\
\hline $\begin{array}{l}\text { Fife et al., } \\
2012^{13}\end{array}$ & $\begin{array}{l}\text { Avaliar e comparar } \\
\text { as mudanças no } \\
\text { volume de edema do } \\
\text { braço, obtidas com } \\
\text { a utilização de um } \\
\text { sistema de dispositivo } \\
\text { de compressão } \\
\text { pneumática avançado } \\
\text { com um sistema } \\
\text { padrão de compressão } \\
\text { pneumática }\end{array}$ & 36 & $\begin{array}{l}\text { Grupo I: } \\
63,9 \\
+-12,2 \\
\text { Grupo II: } \\
59,7 \\
+-12,6\end{array}$ & $\begin{array}{l}\text { Os indivíduos realizaram o tratamento } \\
\text { em casa } 1 \text { h/ dia por } 12 \text { semanas. Todos os } \\
\text { sujeitos utilizaram órtese de compressão } \\
\text { por } 23 \text { h por dia. } 0 \text { grupo I realizou CPI, } \\
\text { que consiste num gradiente de compressão } \\
\text { pneumática sequencial no qual o vestuário } \\
\text { é composto por } 4 \text { câmaras de compressão } \\
\text { que inflam sequencialmente (18 s cada). } \\
\text { A pressão foi definida de acordo com as } \\
\text { instruções do fabricante que sugeriu a } \\
\text { utilização de } 30 \text { mmHg. } 0 \text { grupo II realizou } \\
\text { compressão pneumática avançada } \\
\text { que se consistiu por um controlador } \\
\text { eletrônico formado por } 3 \text { peças que } \\
\text { tratam do membro superior completo, } \\
\text { peito adjacente, e o quadrante troncular. } \\
0 \text { conjunto de vestuário contém } 26-28 \\
\text { câmaras. } 0 \text { ciclo de inflação/deflação para } \\
\text { cada câmara é de } 1-3 \text { s de duração. A } \\
\text { pressão utilizada seguiu a configuração } \\
\text { padrão também sugerida pelo fabricante } \\
\text { que se manteve entre } 9,0+-4,2 H g \text { e } 13,7 \\
+-4,8 \text { mmHg no antebraço }\end{array}$ & $\begin{array}{l}\text { Perimetria } \\
\text { de membro } \\
\text { superior a cada } \\
4 \mathrm{~cm}\end{array}$ & $\begin{array}{l}\text { Houve uma redução de volume } \\
\text { de } 6,3+-216 \mathrm{~mL} \text { para o grupo } \\
\text { I (16\%) e de } 118+\text { - } 170 \\
(29 \%) \text { para o grupo avançado, } \\
\text { apresentando significância } \\
\text { estatística no grupo avançado, } \\
\text { sugerindo que a compressão } \\
\text { pneumática no membro superior, } \\
\text { tronco e peito promove melhores } \\
\text { resultados }\end{array}$ \\
\hline $\begin{array}{l}\text { Gurdal et al., } \\
2012^{14}\end{array}$ & $\begin{array}{l}\text { Avaliar a eficácia de } \\
\text { duas modalidades de } \\
\text { tratamento, sendo } \\
\text { DLM e bandagem } \\
\text { de compressão } \\
\text { combinados } \\
\text { comparados com CPI }\end{array}$ & 30 & $\begin{array}{l}\text { Grupo I: } \\
58,13- \\
10,54 \\
\text { Grupo II: } \\
50,13- \\
10,83\end{array}$ & $\begin{array}{l}\text { Os grupos foram tratados durante } 6 \\
\text { semanas } \\
\text { A CPI foi realizada com uma pressão de } \\
25 \mathrm{mmHg} \\
\text { Grupo I: Foram aplicados DLM e EC } \\
\text { Grupo II: Foram aplicadas autodrenagem } \\
\text { e CPI. Ambos os grupos continuaram o } \\
\text { tratamento com roupas de compressão } \\
\text { exercícios e cuidados com a pele }\end{array}$ & $\begin{array}{l}\text { Perimetria } \\
\text { de membro } \\
\text { superior a cada } \\
10 \mathrm{~cm}\end{array}$ & $\begin{array}{l}\text { A redução no volume total do } \\
\text { braço foi de } 529 \mathrm{~mL}(14,9 \%) \text { e } \\
439 \mathrm{~mL}(12,2 \%) \text { nos grupos I e } \\
\text { II, respectivamente. Não houve } \\
\text { diferença significativa entre } \\
\text { os grupos e ambas as terapias } \\
\text { foram consideradas eficazes para } \\
\text { o tratamento do linfedema de } \\
\text { membro superior }\end{array}$ \\
\hline $\begin{array}{l}\text { Uzkeser } \\
2013^{15}\end{array}$ & $\begin{array}{l}\text { Investigar a eficácia } \\
\text { e a contribuição da } \\
\text { CPI no tratamento do } \\
\text { linfedema e avaliar a } \\
\text { correlação de métodos } \\
\text { de avaliação para o } \\
\text { linfedema }\end{array}$ & 30 & $\begin{array}{l}\text { Grupo I: } \\
56 \text { (37-75) } \\
\text { Grupo II: } \\
55 \\
(42-75)\end{array}$ & $\begin{array}{l}\text { Todos os grupos foram tratados } 5 \text { vezes } \\
\text { por semana durante } 3 \text { semanas (para um } \\
\text { total de } 15 \text { sessões) } \\
0 \text { grupo FCD: cuidados com a pele, } \\
\text { drenagem linfática manual, enfaixamento } \\
\text { funcional, órtese de compressão, e } \\
\text { exercícios } \\
\text { Grupo FCD + CP: CPI + FCD }\end{array}$ & $\begin{array}{l}\text { Método de } \\
\text { deslocamento de } \\
\text { água }\end{array}$ & $\begin{array}{l}\text { Volume inicial do grupo 1: } 630 \\
\text { (180-1,820) mL } \\
\text { Volume final: } 480 \text { (0-1,410) mL } \\
\text { Após } 7 \text { semanas, houve um leve } \\
\text { aumento no volume: } 510 \text { (50-430) } \\
\mathrm{mL} \\
\text { No grupo 2, o volume inicial } 840 \\
(220-3,460) \mathrm{mL} \text {; e depois da } \\
\text { terapia } 500 \text { (60-2,160) mL. Após } 7 \\
\text { semanas, } 500 \text { (180-2,080) mL. } \\
\text { Não houve diferença significativa. } \\
\text { A compressão pneumática não } \\
\text { promove maiores ganhos no } \\
\text { tratamento do linfedema quando } \\
\text { comparada com a DLM }\end{array}$ \\
\hline
\end{tabular}

significativa na redução do volume entre os grupos; ou seja, o ECF mostrou-se eficaz quando utilizado de forma isolada ou associado com a DLM. Para comparar a diferença entre os grupos, os dados foram analisados por meio do teste $t$ de Student e a taxa de reduçáo foi avaliada usando análise de univariância. O nível de significância foi estabelecido com valor de $\mathrm{p}<0,05$.
O estudo realizado por Damstra et al. $(2008)^{17}$ analisou a eficácia da técnica de ECF com nível de pressão alta e baixa. Os autores identificaram que não há diferença estatisticamente significativa entre os dois níveis de pressão utilizados; porém o enfaixamento com menor pressão foi mais bem tolerado pelas pacientes. Os autores utilizaram análises de variância e teste de comparaçôes 
Quadro 2. Característica dos estudos clínicos randomizados selecionados sobre fisioterapia complexa descongestiva e enfaixamento compressivo

\begin{tabular}{|c|c|c|c|c|c|c|}
\hline Estudo/Ano & Objetivo & N & $\begin{array}{l}\text { Média de } \\
\text { idade }\end{array}$ & Tratamento & $\begin{array}{c}\text { Avaliação do } \\
\text { linfedema }\end{array}$ & Resultados obtidos \\
\hline $\begin{array}{l}\text { McNeely et } \\
\text { al., } 2004^{16}\end{array}$ & $\begin{array}{l}\text { Comparar o tratamento } \\
\text { com DLM asociado ao } \\
\text { EC com EC sozinho, na } \\
\text { redução do linfedema } \\
\text { de membro superior }\end{array}$ & 50 & $\begin{array}{l}59 \text { anos } \\
+-13 \text { anos }\end{array}$ & $\begin{array}{l}\text { Todos os participantes receberam } 4 \\
\text { semanas de tratamento, com aplicação } \\
\text { de DLM, de segunda a sexta feira. Um } \\
\text { grupo recebeu } 45 \text { minutos de DLM e EC } \\
\text { com maior pressão distal, reduzindo } \\
\text { para proximal, utilizando uma camada } \\
\text { de algodão; uma camada de espuma; e, } \\
\text { após, enfaixamento em forma de oito } \\
0 \text { grupo que recebeu apenas EC seguiu o } \\
\text { mesmo protocolo do que realizou a DLM }\end{array}$ & $\begin{array}{l}\text { Método de } \\
\text { imersão } \\
\text { em água e } \\
\text { perimentria a } \\
\text { cada } 4 \mathrm{~cm}\end{array}$ & $\begin{array}{l}\text { Os dois grupos reduziram } \\
\text { significamente o volume em } 4 \\
\text { semanas. Grupo DLM reduziu de } \\
695 \mathrm{~mL} \text { para } 435 \mathrm{~mL} \text {, totalizando } \\
46,1 \% \text { de redução, enquanto o } \\
\text { grupo } \mathrm{EC} \text { reduziu de } 672 \mathrm{~mL} \text { para } \\
426 \mathrm{~mL} \text {, diminuindo } 38,6 \% \text { o volume } \\
\text { quando avaliado por deslocamento de } \\
\text { água em imersão em um recepiente } \\
\text { preestabelecido. Já quando o volume } \\
\text { foi avaliado por fórmula matemática } \\
\text { a partir da perimetria, os grupos } \\
\text { reduziram } 44,1 \% \text { no grupo DLM (de } \\
666 \text { para } 425 \mathrm{~mL} \text { ) e } 37,2 \% \text { no grupo } \\
\text { EC (656 para } 412 \mathrm{~mL} \text { ). Nenhuma } \\
\text { diferença significativa foi encontrada } \\
\text { entre os grupos }\end{array}$ \\
\hline $\begin{array}{l}\text { Damstra et } \\
\text { al., } 2008^{17}\end{array}$ & $\begin{array}{l}\text { Determinar se existe } \\
\text { diferença entre } 0 \\
\text { EC com baixa e alta } \\
\text { pressão na redução do } \\
\text { linfedema de membro } \\
\text { superior }\end{array}$ & 36 & $\begin{array}{l}\text { Grupo A: } \\
60,5 \text { (45-84 } \\
\text { years) } \\
\text { Grupo B: } \\
61,2 \text { (50-73 } \\
\text { years) }\end{array}$ & $\begin{array}{l}\text { Todos os participantes do estudo } \\
\text { receberam EC. Houve aplicação de sondas } \\
\text { para aferição de pressão para que se } \\
\text { enfaixassem os braços em pressões } \\
\text { cuidadosamente preestabelecidas (baixa } \\
\text { pressão: } 20-30 \mathrm{mmHg} \text {; alta pressão: } \\
44-58 \mathrm{mmHg} \text { ). A redução do volume de } \\
\text { curto prazo foi medida em duas horas, } \\
\text { o enfaixamento removido e um novo } \\
\text { realizado permanecendo por } 24 \text { horas, } \\
\text { utilizando a pressão } \\
0 \text { efeito mensurado foi agudo, } 2 \text { e } 24 \\
\text { horas após aplicação da bandagem }\end{array}$ & $\begin{array}{l}\text { Método de } \\
\text { imersão em } \\
\text { água }\end{array}$ & $\begin{array}{l}\text { A redução média de volume do } \\
\text { membro superior no grupo que } \\
\text { utilizou enfaixamento de baixa } \\
\text { pressão foi de } 104,5 \mathrm{~mL} \text { após } 2 \\
\text { horas; e } 217 \mathrm{~mL} \text { após } 24 \text { horas. } 0 \\
\text { grupo que utilizou alta pressão no } \\
\text { enfaixamento teve uma redução } \\
\text { de } 56,5 \mathrm{~mL} \text { após duas horas e } \\
167,5 \mathrm{~mL} \text { após } 24 \text { horas. Apesar de } \\
\text { ficar evidente que } 0 \text { enfaixamento } \\
\text { com baixa pressão produziu uma } \\
\text { maior redução no volume, não } \\
\text { houve diferença estatisticamente } \\
\text { significante entre resultados } \\
\text { obtidos nos grupos A e B. Além } \\
\text { disso, o enfaixamento de baixa } \\
\text { pressão foi mais bem tolerado pelos } \\
\text { participantes }\end{array}$ \\
\hline $\begin{array}{l}\text { Kasseroller et } \\
\text { al., } 2009^{18}\end{array}$ & $\begin{array}{l}\text { Determinar se há } \\
\text { diferença entre o EC } \\
\text { comum e o embebido } \\
\text { em solução de } \\
\text { alginato na redução } \\
\text { e/ou manutenção do } \\
\text { linfedema de membro } \\
\text { superior; verificar } \\
\text { a tolerância dos } \\
\text { pacientes com relação } \\
\text { ao enfaixamento }\end{array}$ & 61 & $\begin{array}{l}57,4 \text { anos } \\
(<28 ; \\
>81)\end{array}$ & $\begin{array}{l}\text { De segunda a quinta-feira, os dois grupos } \\
\text { receberam o mesmo tratamento: } 90 \text { a } 120 \\
\text { minutos de DLM, compressão pneumática } \\
\text { e cinesioterapia } \\
\text { De Sexta-feira a domingo: } \\
\text { Grupo A: Enfaixamento convencional } \\
\text { Grupo B: Enfaixamento com bandagem } \\
\text { embebida em solução de alginato. No } \\
\text { total, foram } 22 \text { dias de tratamento (3 } \\
\text { finais de semana no hospital) }\end{array}$ & $\begin{array}{l}\text { Perimetria } \\
\text { de membro } \\
\text { superior a cada } \\
4 \mathrm{~cm}\end{array}$ & $\begin{array}{l}\text { Redução média do volume de } \\
264,5 \mathrm{~mL}+-174,482(8,63 \% \\
+-4,960) \text { no grupo } \mathrm{A} \mathrm{e} 322,5 \mathrm{~mL} \\
+-139,480(10,50 \%+-4,433) \text { no } \\
\text { grupo B } \\
\text { A diferença entre os dois grupos } \\
\text { não foi significativa; porém o } \\
\text { enfaixamento com alginato foi } \\
\text { considerado mais confortável pelas } \\
\text { pacientes }\end{array}$ \\
\hline $\begin{array}{l}\text { King et al., } \\
2011^{19}\end{array}$ & $\begin{array}{l}\text { Comparar os efeitos } \\
\text { da utilização de } \\
\text { órteses de compressão } \\
\text { com o } \mathrm{EC} \text { na fase } \\
\text { inicial de aplicação } \\
\text { de um protocolo } \\
\text { de FCD no volume } \\
\text { do membro, nos } \\
\text { sintomas relacionados } \\
\text { ao linfedema (dor, } \\
\text { sensação de peso, e } \\
\text { tensão), e no prejuízo } \\
\text { funcional }\end{array}$ & 21 & $\begin{array}{l}\text { Grupo 1: } \\
57(44-69) \\
\text { Grupo } \\
2: 64,5 \\
(52-76)\end{array}$ & $\begin{array}{l}\text { Todos osparticipantes receberam } 10 \\
\text { sessões de FCD: DLM seguido de cuidados } \\
\text { com a pele, órtese de compressão elástica / } \\
\text { enfaixamento e exercícios. } 0 \text { grupo } 1 \\
\text { utilizou órtese de compressão elástica e } \\
\text { o grupo } 2 \text { utilizou enfaixamento durante } \\
\text { o dia e à noite. Ao final, todos pacientes } \\
\text { utilizaram órtese compressiva elástica }\end{array}$ & $\begin{array}{l}\text { Perimetria } \\
\text { de membro } \\
\text { superior a cada } \\
4 \text { cm e método } \\
\text { de deslocamento } \\
\text { de água }\end{array}$ & $\begin{array}{l}0 \text { grupo } \mathrm{EC} \text { reduziu mais o volume } \\
\text { (-70 vs }-5 \mathrm{~mL} \text { no décimo dia de } \\
\text { intervenção; }-97,5 \text { vs }-50 \mathrm{~mL} \text { em } \\
3 \text { meses); porém houve piora } \\
\text { funcional nesse grupo. Não } \\
\text { houve diferença significativa } \\
\text { observada entre os dois métodos } \\
\text { de compressão com relação à dor, } \\
\text { sensação de peso e de tensão }\end{array}$ \\
\hline
\end{tabular}


múltiplas de Tukey para comparar os valores iniciais de pressão e volume em relação aos valores subsequentes dentro dos grupos. As comparaçóes entre os dois grupos de tratamento foram realizadas utilizando o teste de Mann Whitney-não-paramétrico. Ainda foi realizada uma comparação de pressão e perda de volume, utilizando a correlação náo paramétrica pelo coeficiente de Spearman. O nível de significância foi aceito em $\mathrm{p}<0,05$.

Kasseroller et al. (2009) ${ }^{18}$ compararam a aplicação do ECF tradicional com ECF utilizando bandagens embebidas em alginato. Essa soluçáo é feita à base de algas marinhas marrons, fazendo com que a bandagem se torne semirrígida após a secagem. Ambos os grupos também realizaram DLM, CPI e cinesioterapia. O grupo que utilizou bandagem embebida em alginato apresentou maior redução no volume do linfedema; porém não houve diferença estaticamente significativa entre os dois grupos. A comparação estatística entre os grupos foi realizada utilizando-se análise de variância univariada (ANOVA), e o nível de significância foi estabelecido para $\mathrm{p}<0,05$.

Por fim, King et al. (2011) ${ }^{19}$ compararam o uso de ECF com órteses de compressão e identificaram que o grupo que recebeu ECF reduziu mais o volume; porém houve uma piora na funcionalidade do membro superior no grupo enfaixado. Foi utilizada estatística descritiva para descrever as diferenças nas avaliaçóes volumétricas e na pontuação de um questionário aplicado para avaliar a funcionalidade do membro superior. As diferenças entre grupos foram comparadas pelo teste de Wilcoxon.

\section{FISIOTERAPIA COMPLEXA DESCONGESTIVA ASSOCIADA AO KINESIOTAPE}

Com o intuito de verificar a influência da K-TAPE na redução do linfedema de membro superior pós-mastectomia, foi localizado um ensaio clínico randomizado (Quadro 3).
Tsai et al. (2009)20 compararam os efeitos da FCD associada com ECF e FCD, substituindo o enfaixamento pela K-TAPE, ambos associados à CPI. O estudo avaliou e tratou 41 participantes com linfedema de membro superior pós-mastectomia por quatro semanas e verificou que houve reduçáo no volume do membro afetado em ambos os grupos, sem diferença estatisticamente significativa, sendo a K-TAPE melhor aceita pelas participantes, gerando melhor conforto. Os autores utilizaram teste $t$ de Student para amostras independentes, teste de Mann-Whitney-U e qui-quadrado para analisar as variáveis de desfecho na linha de base e ao final de tratamento. O nível de significância estatística foi estabelecido em $\mathrm{p}<0,05$.

\section{DISCUSSÃO}

Todos os estudos incluídos nesta revisão sistemática encontraram resultados positivos com a utilização da terapia por compressão externa, na reduçáo do volume de linfedema, mesmo realizando protocolos e técnicas diferenciadas ${ }^{11-20}$ sendo o ECF, a K-TAPE e a compressão pneumática, associados à $\mathrm{FCD}$, considerados eficazes na reduçáo do linfedema de membro superior; porém esses estudos se mostram divergentes quanto à superioridade de um ou outro modo.

O linfedema de membro superior pode ser controlado; porém, não curado ${ }^{4}$. A FCD atualmente é o tratamento preconizado pela Sociedade Internacional de Linfologia para essa disfunção ${ }^{21}$. Diferentes autores afirmam que esse é o método mais eficiente para a redução do linfedema, constituído por diferentes técnicas como exercícios descongestionantes, cuidados com a pele, drenagem linfática manual, além de técnicas de compressão externa que constituem parte importante da terapia para reabsorção linfática ${ }^{7,21-22}$.

$\mathrm{O}$ EC não só mantém como incrementa a absorção linfática e, em conjunto com a cinesioterapia, estimula o

Quadro 3. Ensaio clínico randomizado: tratamento oferecido e resultados obtidos pelos autores

\begin{tabular}{|c|c|c|c|c|c|c|}
\hline Estudo/Ano & Objetivo & $\mathbf{N}$ & $\begin{array}{l}\text { Média de } \\
\text { idade }\end{array}$ & Tratamento & $\begin{array}{l}\text { Avaliação do } \\
\text { linfedema }\end{array}$ & Resultados \\
\hline $\begin{array}{l}\text { Tsai et al., } \\
2009^{20}\end{array}$ & $\begin{array}{l}\text { Comparar os efeitos da } \\
\text { FCD associada com EC } \\
\text { e FCD, substituindo } \\
\text { o enfaixamento } \\
\text { pela K-TAPE, } \\
\text { ambos associados } \\
\text { à compressão } \\
\text { pneumática }\end{array}$ & 41 & $\begin{array}{l}54,6 \text { anos } \\
(<36 \\
>75)\end{array}$ & $\begin{array}{l}\text { A intervenção teve a duração de } 4 \\
\text { semanas. Um grupo recebeu EC, } 30 \\
\text { minutos de DLM e } 1 \text { hora de compressão } \\
\text { pneumática, cinesioterapia e orientações } \\
\text { sobre cuidados com a pele. } 0 \text { outro grupo } \\
\text { recebeu DLM combinada com compressão } \\
\text { pneumática, aplicação da K-TAPE ao invés } \\
\text { do EC }\end{array}$ & $\begin{array}{l}\text { Método de } \\
\text { imersão } \\
\text { em água e } \\
\text { perimetria } \\
\text { de membro } \\
\text { superior a cada } \\
3 \mathrm{~cm}\end{array}$ & $\begin{array}{l}\text { Houve redução de 84,0mL no volume } \\
\text { do membro superior no grupo que } \\
\text { realizou enfaixamento e de } 51,3 \mathrm{~mL} \\
\text { no grupo que utilizou a K-TAPE. } \\
\text { A aceitação da K-TAPE foi melhor do } \\
\text { que o enfaixamento. Os participantes } \\
\text { relataram que a K-TAPE gera maior } \\
\text { conforto e comodidade em } \\
\text { atividades diárias. No entanto, } \\
\text { as participantes do grupo K-TAPE } \\
\text { apresentaram mais lesões cutâneas } \\
\text { no membro superior quando } \\
\text { comparadas ao grupo que realizou } 0 \\
\text { enfaixamento }\end{array}$ \\
\hline
\end{tabular}


funcionamento linfático ${ }^{4}$; porém, cabe ressaltar que apesar de essa técnica se mostrar eficaz, os estudos analisados não comprovam que a sua utilizaçáo altera a resposta ao tratamento quando comparado a outras ténicas.

Já a K-TAPE tem a propriedade de melhorar o fluxo linfático por produzir diferentes pressóes na pele, favorecendo o bombeamento para as regióes com menos pressão e por sua vantagem de permanecer na pele por dias; e, por promover maior conforto quando o membro está em repouso, pode aumentar a aderência das pacientes à terapêutica9.

Essa técnica surge como uma alternativa ao ECF; porém, os estudos existentes são escassos e, em sua maioria, apresentam um número pequeno de sujeitos. Seu uso vem sendo discutido em diversas áreas, inclusive na oncologia. Tsai et al. em $2009^{20}$ constataram que não há diferença significativa entre o uso do EC e da K-TAPE na redução do volume do membro acometido, mas essa técnica pode ser utilizada como alternativa na fase intensiva de reabilitação para pacientes que apresentem qualquer tipo de contraindicação para o EC.

Além do tratamento do linfedema, as técnicas do EC e a K-TAPE se propóem a manter por maior tempo possível a redução do linfedema obtida pela FCD. A não utilizaçáo da bandagem compressiva ou braçadeira elástica associada à FCD foram fatores de risco para um aumento da gravidade do linfedema ${ }^{23}$. Algumas pacientes têm restrição em utilizar o EC devido ao clima quente e úmido, prejudicando assim os efeitos da terapia ${ }^{20}$, o que se pode aplicar ao público brasileiro, devido às condições climáticas. Além disso, o EC pode ser demorado e difícil de realizar para pacientes que apresentem limitações físicas ou comorbidades ${ }^{13}$.

A compressão pneumática também é uma modalidade comumente utilizada no tratamento do linfedema, auxiliando a drenagem dos fluidos para as áreas proximais pelo aumento das pressóes nos vasos linfáticos e meio intersticial, por meio de câmaras de ar com vários formatos, por um sistema de compressão de ar, e é de fácil aplicação, podendo a própria paciente realizar a técnica ${ }^{21}$. No entanto, os estudos que abordam essa técnica mostram-se controversos quando comparados com outras terapêuticas descongestivas, na medida em que a sua utilização nem sempre produz resultados melhores.

A literatura preconiza que as pressóes utilizadas durante a compressão pneumática se mantenham entre 40 e $60 \mathrm{mmHg}$ e que o tempo máximo de aplicação seja de 30 minutos para evitar lesóes nos vasos linfáticos e no membro em tratamento ${ }^{4,21}$. Os estudos aqui revisados mantiveram seus níveis de pressão de 25 a $50 \mathrm{mmHg}$, não ultrapassando os valores previstos para evitar lesão; porém três estudos realizaram uma aplicação maior do que 30 minutos $^{13-15}$. Não houve evidência de lesão ou de resultados insatisfatórios nos estudo que realizaram a intervenção por tempo maior do que o indicado na literatura.

\section{CONCLUSÃO}

De um modo geral, os estudos analisados nessa revisão sistemática demonstraram que o ECF, a CPI e a K-TAPE, amplamente utilizados na prática clínica, se mostraram eficientes na reduçáo do linfedema de membro superior em mulheres mastectomizadas; porém, quando as técnicas são comparadas entre si, não é possível afirmar qual delas se mostra mais eficaz na reduçáo do linfedema. Sugerem-se, portanto, novos estudos clínicos randomizados que busquem elucidar os efeitos produzidos por essas técnicas, principalmente no que se refere à K-TAPE, cujas pesquisas ainda se mostram incipientes.

\section{CONTRIBUIÇÕES}

Suiane Weimer Cendron trabalhou em todas as fases do estudo. Luciana Laureano Paiva trabalhou na metodologia, redação e revisão final do estudo. Caroline Darski e Cássia Colla trabalharam na pesquisa e na metodologia.

\section{Declaraçáo de Conflito de Interesses: Nada a Declarar.}

\section{REFERÊNCIAS}

1. Instituto Nacional do Câncer José Alencar Gomes da Silva. Estimativa 2014: incidência do câncer no Brasil [Internet]. Rio de Janeiro: Inca; 2014. [citado em 2015 mar.]. Disponível em http://www. inca. gov. br/ estimativa/2014/.

2. Bregagnol RK, Dias AS. Alterações funcionais em mulheres submetidas à cirurgia de mama com linfadenectomia axilar total. Rev Bras Cancerol. 2010;56(1):25-33.

3. Tacani PM, Camargo RAL, Silva G, Moreira BC, Batista PA N, Montezello D, et al. Fisioterapia descongestiva no linfedema de membros superiores pós-mastectomia: estudo retrospectivo. Revista Brasileira de Ciências da Saúde. 2013; 37(3):17-23.

4. Leal NFBS, Carrara HHA, Vieira KF, Ferreira CHJ. Tratamentos fisioterapêuticos para o linfedema póscâncer de mama: uma revisão de literatura. Rev LatinoAm Enfermagem. 2009;17(5):730-6.

5. Oliveira J, César TB. Influência da fisioterapia complexa descongestiva associada à ingestão de triglicerídeos de cadeia média no tratamento do linfedema de membro superior. Rev Bras Fisioter. 2008;12(1):31-6

6. Luz ND, Lima ACG. Recursos fisioterapêuticos em linfedema pós-mastectomia: uma revisão de literatura. Fisiotermov. 2011;24(1):191-200.

7. Godoy JMP, Godoy MFG. Drenagem linfática manual: novo conceito. J. Vasc. Br. 2004;3(1):77-80. 
8. Vieiros I, Nunes R, Martins F. Complicações da mastectomia: linfedema de membro superior. Acta Med Port. 2007;20:335-40.

9. Navarro-Brazález B, Sánchez-Sanchez B. El vendaje en el tratamiento fisioterapéutico del linfedema secundario a cancer de mama: una serie de casos. Fisioterapia. 2014;36(1):49-53.

10. Moher D, Liberati A, Tetzlaff J, Altman DG, PRISMA Group. Reprint - preferred reporting items for systematic reviews and meta-analyses: the PRISMA statement. Phys Ther. 2009;89(9):873-80

11. Szolnoky G, Lakatos B, Keskeny T, Varga E, Varga M, Dobozy A, et. al.. Intermittent pneumatic compression acts synergistically with manual lymphatic drainage in complex decongestive physiotherapy for breast cancer treatment-related lymphedema. Lymphology. 2009;42(4):188-94.

12. Haghighat SM, Lotfi-Tokaldany M, Yunesian M, Akbari ME, Nazemi F, Weiss J. Comparing two treatment methods for post mastectomy lymphedema: complex decongestive therapy alone and in combination with intermittent pneumatic compression. Lymphology. 2010; 43(1):25-33

13. Fife CE, Davey S, Maus EA, Guilliod R, Mayrovitz HN. A randomized controlled trial comparing two types of pneumatic compression for breast cancer-related lymphedema treatment in the home. Support Care Cancer. 2012;20(12):3279-86.

14. Gurdal SO, Kostanoglu A, Cavdar I, Ozbas A, Cabioglu $\mathrm{N}$, Ozcinar B, et al. Comparison of intermittent pneumatic compression with manual lymphatic drainage for treatment of breast cancer-related lymphedema. Lymphat Res Biol 2012;10(3):129-35.

15. Uzkeser H, Karatay S, Erdemci B, Koc M, Senel K. Efficacy of manual lymphatic drainage and intermittent pneumatic compression pump use in the treatment of lymphedema after mastectomy: a randomized controlled trial. Breast Cancer. 2013 [Epub ahead of print].

16. McNeely ML, Magee DJ, Lees AW, Bagnall KM, Haykowsky M, Hanson J. The addition of manual lymph drainage to compression therapy for breast cancer related lymphedema: a randomized controlled trial. Breast Cancer Res Treat. 2004;86(2):95-106.

17. Damstra RJ, Partsch H. Compression therapy in breast cancer-related lymphedema: A randomized, controlled comparative study of relation between volume and interface pressure changes. J Vasc Surg. 2009;49(5):1256-63.

18. Kasseroller RG, Brenner E. A prospective randomised study of alginate-drenched low stretch bandages as an alternative to conventional lymphologic compression bandaging. Support Care Cancer. 2010;18(3):343-50.

19. King M, Deveaux A, White H, Rayson D. Compression garments versus compression bandaging in decongestive lymphatic therapy for breast cancer-related lymphedema: a randomized controlled trial. Support Care Cancer. 2012;20(5): 1031-6.

20. Tsai HJ, Hung HC, Yang JL, Huang CS, Tsauo JY. Could Kinesio tape replace the bandage in decongestive lymphatic therapy for breast-cancerrelated lymphedema? A pilot study. Support Care Cancer. 2009; 17(11):1353-60.

21. Rech JBS, Nóbrega L, Lemos A. Compressão pneumática no tratamento de linfedema pós-mastectomia: revisão sistemática. Rev Bras Cancerol. 2010;56(4):483-91

22. Santos DA, Cipolla LV, Oliveira MMF. Atuação da fisioterapia no tratamento do linfedema após câncer de mama. Ensaios e Ciência: Ciências Biológicas, Agrárias e da Saúde. 2010;14(1):177-86

23. Vignes S, Porcher R, Arrault M, Dupuy A. Long-term management of breast cancer- related lymphedema after intensive decongestive physiotherapy. Breast Cancer Res Treat. 2007;101(3): 285-90. 


\section{Abstract}

Introduction: Breast cancer is the most frequent cancer in the female population, and the lymphedema of the upper limb the most frequent complication in mastectomy. Object: To perform a systematic review and assess the effectiveness of complex decongestive physical therapy associated with the use of kinesiotape, compressive bandaging and pneumatic compression in the treatment of secondary lymphedema in breast cancer. Method: A systematic review the following databases was performed: MEDLINE, PEDro, Cochrane CENTRAL, EMBASE, Portal Periódicos Capes, CINAHL and Google Scholar. The keywords were: "kinesiotape", "bandage", "drainage", "mastectomy", "lymphedema", "physioterapy", "physical therapy", "compressive bandaging", "pneumatic compression". Results: We selected ten randomized controlled trials. It was verified in studies that pneumatic compression reduces the volume of lymphedema when associated with complex decongestive physiotherapy. The compressive bandaging proved to be effective in reducing lymphedema, but is often abandoned by patients during treatment due to discomfort. The kinesiotape is an alternative therapy for patients who aren't adapted to the bandaging. However, studies that prove its effectiveness are mostly case studies and, for that reason, are not sufficient to confirm its clinical applicability. Conclusion: This systematic review has proved to be important in the sense that it allowed us to analyze the effects of each technique. However, randomized clinical trials are still scarce on this topic, especially on the use of kinesiotape, making it difficult to establish which technique is more effective in reducing lymphedema and maintenance of reduction levels achieved during complex decongestive physiotherapy.

Key words: Mastectomy; Lymphedema; Physioterapy; Compression Bandages

\section{Resumen}

Introducción: El cáncer de mama es el cáncer más frecuente en la población femenina, y el linfedema del miembro superior es la complicación más frecuente en el de la mastectomía. Objetivo: Evaluar la efectividad de la terapia física descongestiva compleja asociada con el uso de kinesiotape, vendaje de compresión y la compresión neumática en el tratamiento del linfedema secundario al cáncer de mama. Método: Revisión sistemática, se utilizaron las siguientes bases de datos: MEDLINE, PEDro, Cochrane CENTRAL, EMBASE, Portal Periódicos Capes, Cinahl y Google Académico. Las palabras clave fueron: "kinesiotape", "bandage", "drainage", "mastectomy", "lymphedema", "physioterapy", "physical therapy", "compressive bandaging", "pneumatic compression”. Resultados: Se seleccionaron diez estudios aleatorios. Se comprobó en los estudios que la compresión neumática reduce los niveles de volumen del linfedema cuando se asocia con la fisioterapia descongestiva compleja. El vendaje compresivo se muestra eficaz para reducir el linfedema, pero es abandonada por los pacientes durante el tratamiento porque causa molestias. El kinesiotape es una terapia alternativa para pacientes que no se han adaptado al vendaje. Sin embargo, los artículos que demuestran su eficacia en su mayoría son estudios de casos y por esta razón no son suficientes para confirmar su aplicabilidad clínica. Conclusión: Esta revisión sistemática ha demostrado ser importante, ya que nos permitió analizar los efectos de cada una de las técnicas. Sin embargo, los ensayos clínicos aleatorizados son escasos en este tema, especialmente en el uso de kinesiotape, por esto es difícil establecer cuál es la técnica más eficaz para reducir el linfedema y el mantenimiento de los niveles de reducción alcanzados durante la fisioterapia descongestiva compleja.

Palabras clave: Mastectomía; Linfedema; Fisioterapia; Vendajes de Compresión 